\title{
Inferences on a Scale Parameter of Bivariate Rayleigh Distribution by Ranked Set Sampling
}

\author{
Saeid Tahmasebi \\ Department of Statistics, Persian Gulf University, Bushehr, Iran \\ tahmasebi@pgu.ac.ir \\ Maryam Eskandarzadeh \\ Department of Statistics, Persian Gulf University, Bushehr, Iran \\ stahmasby@yahoo.com \\ Zahra Almaspoor \\ Department of Statistics, Persian Gulf University, Bushehr, Iran \\ s.tahmasebi57@gmail.com
}

\begin{abstract}
In this paper, we obtain several estimators of a scale parameter of Morgenstern type bivariate Rayleigh distribution based on the observations made on the units of the ranked set sampling regarding the study variable which is correlated with the auxiliary variable. We also compare the efficiency of these estimators. Finally, we illustrate the methods developed by using a real data set.
\end{abstract}

Keywords: Best linear unbiased estimator, Double robust extreme ranked set sampling, Extreme ranked set sampling, Ranked set sampling, Relative efficiency, Rayleigh distribution.

\section{Introduction}

Morgenstern (1956) defined a class of bivariate distributions, and Farlie (1960) extend it to the multivariate case. This class of distributions is known as Farlie-GumbelMorgenstern (FGM) distribution. Some well-known marginal distributions are considered and studied in literature: For example logistic (Gumbel, 1961), gamma (D'Este, 1981 ; Tahmasebi and Jafari, 2015), uniform (Bairamov and Bekci, 1999 ; Tahmasebi and Jafari, 2012; Singh and Mehta, 2015), exponential (Gumbel, 1960, Balasubramanian and Beg, 1997; Chacko and Thomas, 2008, 2011) and generalized exponential (Tahmasebi and Jafari, 2014, 2015) distributions. A new member of bivariate FGM distribution is Morgenstern type bivariate Rayleigh distribution (MTBRD) with the cumulative distribution function (cdf) as

$$
F_{X, Y}(x, y)=\left(1-e^{-\frac{x^{2}}{2 \sigma_{1}^{2}}}\right)\left(1-e^{-\frac{y^{2}}{2 \sigma_{2}^{2}}}\right)\left(1+\alpha e^{-\frac{x^{2}}{2 \sigma_{1}^{2}}-\frac{y^{2}}{2 \sigma_{2}^{2}}}\right), \quad x, y>0,
$$

and the probability density function (pdf) as

$$
f_{X, Y}(x, y)=\frac{x y}{\sigma_{1}^{2} \sigma_{2}^{2}} e^{-\frac{x^{2}}{2 \sigma_{1}^{2}}-\frac{y^{2}}{2 \sigma_{2}^{2}}}\left[1+\alpha\left(2 e^{-\frac{x^{2}}{2 \sigma_{1}^{2}}}-1\right)\left(2 e^{-\frac{y^{2}}{2 \sigma_{2}^{2}}}-1\right)\right] .
$$

We consider several unbiased estimators of parameter $\sigma_{2}$ using ranked set sampling (RSS). This technique of sampling was first proposed by McIntyre (1952) and has a more efficient sampling method than simple random sampling (SRS) method for estimating the population mean. Some modifications of RSS are presented in literatures: For example 
modified ranked set sampling procedure by Stokes (1980), extreme ranked set samples (ERSS) by Samawi et al. (1996), moving extreme ranked set sampling (MERSS) by AlOdat and Al-Saleh (2001), and double robust extreme ranked set sampling (DRERSS) by Al-Omari (2011).

RSS are applied for estimating parameters of some distributions: for example locationscale family of distribution by Stokes (1995), two-parameter exponential distribution by Lam et al. (1994), bivariate normal distribution by Al-Saleh and Al-Ananbeh (2005,2007), Morgenstern type bivariate exponential distribution by Chacko and Thomas (2008), Downton's bivariate exponential distribution by Al-Saleh and Diab (2009), and Morgenstern type bivariate gamma distribution by Tahmasebi and Jafari (2015). In this paper we are trying to estimate the mean of the population, under a situation where in measurement of observations are strenuous and expensive.

The organization of this article is as follows. In Section 2, we present three estimators for the scale parameter, $\sigma_{2}$ in MTBRD based on the RSS, and compared the efficiency of these estimators and the estimator based on SRS. In Section 3, we obtain different estimators for $\sigma_{2}$ in MTBRD by using ERSS and MERSS methods. Also, the efficiency of all estimators are evaluated. In Section 4, we obtain unbiased estimator for $\sigma_{2}$ in MTBRD by DRERSS method. In Section 5, we illustrate the proposed methods using a real data set.

\section{Estimating based on RSS}

In the RSS technique, the sample selection procedure is composed of two stages. At the first stage of sample selection, $n$ simple random samples of size $n$ are drawn from an infinite population and each sample is called a set. Then, each of units is ranked from the smallest to the largest. At the second stage, the ${ }_{r}$ th observation unit from the ${ }_{r}$ th ranked set is taken. Ranking of the units is done with a low-level measurement such as using previous experiences, visual measurement or using a concomitant variable. Stokes (1977) described the procedure of RSS for bivariate random variable $(X, Y)$, where $X$ is the variable of interest and $Y$ is a concomitant variable that is not of direct interest but is relatively easy to measure, as follows:

Step 1. Randomly select $n$ independent bivariate samples, each of size $n$.

Step 2. Rank the units within each sample with respect to a variable of interest $X$ together with the $Y$ variate associated.

Step 3. In the ${ }_{r}$ th sample of size $n$, select the unit $\left(X_{(r) r}, Y_{[r] r}\right), r=1,2, \ldots, n$, where $X_{(r) r}$ is the observation measured on the variable $X$ in the ${ }_{r}$ th unit of the RSS and $Y_{[r] r}$ is the corresponding measurement made on the study variable $Y$ of the same unit.

Suppose that the random variable $(X, Y)$ has a MTBRD as defined in (1.1). Let $Y_{[r] r}$, $r=1,2,3, \ldots, n$, be the RSS observations made on the units of the ranked set sampling regarding the study variable $Y$ which is correlated with the auxiliary variable $X$. It is 
clear that $Y_{[r] r}$ is the concomitant of ${ }_{r}$ th order statistic arising from the ${ }_{r}$ th sample. From Scaria and Nair (1999), the pdf of $Y_{[r] r}$ is given by

$$
g_{[r] r}(y)=\frac{y}{\sigma_{2}^{2}} e^{-\frac{y^{2}}{2 \sigma_{2}^{2}}}\left[1+\delta_{r}\left(2 e^{-\frac{y^{2}}{2 \sigma_{2}^{2}}}-1\right)\right], \quad y>0
$$

where $\delta_{r}=\frac{\alpha(n-2 r+1)}{n+1}$. Also, the pdf of $X_{(r) r}$ is

$$
f_{r}(x)=\frac{n !}{(r-1) !(n-r) !} \times \frac{x}{\sigma_{1}^{2}} e^{-\frac{(n-r+1) x^{2}}{2 \sigma_{1}^{2}}}\left[1-e^{-\frac{x^{2}}{2 \sigma_{1}^{2}}}\right]^{r-1} .
$$

Therefore, the mean and variance of $Y_{[r] r}$ are given as

$$
E\left[Y_{[r] r}\right]=\sigma_{2} \beta_{r}, \quad \operatorname{Var}\left[Y_{[r] r}\right]=\sigma_{2}^{2} \lambda_{r}
$$

where $\beta_{r}=\sqrt{\frac{\pi}{2}}+\delta_{r} \frac{\sqrt{\pi}}{2}(1-\sqrt{2})$ and $\lambda_{r}=\frac{4-\pi}{2}-\frac{\pi \delta_{r}^{2}(1-\sqrt{2})^{2}}{4}-\delta_{r}\left[1+\frac{\pi(\sqrt{2}-2)}{2}\right]$.

Theorem 2.1 When $\alpha$ is known, an unbiased estimator for $\sigma_{2}$ based on RSS is

$$
\hat{\sigma}_{2, \mathrm{RSS}}=\frac{1}{n \sqrt{\frac{\pi}{2}}} \sum_{r=1}^{n} Y_{[r] r}
$$

with the variance

$$
\operatorname{Var}\left(\hat{\sigma}_{2, \mathrm{RSS}}\right)=\frac{\sigma_{2}^{2}(4-\pi)}{n \pi}\left(1-b_{n}\right)
$$

where $b_{n}=\frac{\pi(3-2 \sqrt{2})(n-1)}{6(4-\pi)(n+1)} \alpha^{2}$.

Proof. Since $\sum_{r=1}^{n} \delta_{r}=0$, and using (2.3) the proof is obvious.

The random variable $Y$ has a Rayleigh distribution with scale parameter $\sigma_{2}$. Therefore, an unbiased estimator of $\sigma_{2}$ based on a simple random sample (SRS) of size $n$ from Rayleigh distribution is $\hat{\sigma}_{2, \mathrm{SRS}}=\bar{Y} / \sqrt{\frac{\pi}{2}}$ with variance $\frac{\sigma_{2}^{2}(4-\pi)}{n \pi}$. The relative efficiency of $\hat{\sigma}_{2, \mathrm{RSS}}$ to $\hat{\sigma}_{2, \mathrm{SRS}}$ is

$$
e_{1}=e\left(\hat{\sigma}_{2, \mathrm{RSS}} \mid \hat{\sigma}_{2, \mathrm{SRS}}\right)=\frac{\operatorname{Var}\left(\hat{\sigma}_{2, \mathrm{SRS}}\right)}{\operatorname{Var}\left(\hat{\sigma}_{2, \mathrm{RSS}}\right)}=\frac{1}{1-b_{n}} .
$$

Note that $1 \leq e_{1} \leq \frac{10}{9}$. Thus, $\hat{\sigma}_{2, \mathrm{RSS}}$ is more efficient than $\hat{\sigma}_{2, \mathrm{SRS}}$.

Now, we study the efficiency of $\hat{\sigma}_{2, \mathrm{RsS}}$ relative to the best linear unbiased estimator (BLUE) of $\sigma_{2}$, based on $Y_{[r] r}, r=1,2,3, \ldots, n$ of MTBRD, when $\alpha$ is known. Suppose 
that $\mathbf{Y}_{[n]}=\left(Y_{[1] 1}, Y_{[2] 2}, \ldots, Y_{[n] n}\right)^{\prime}$. Then BLUE of $\sigma_{2}$ is derived as ( see David and Nagaraja, 2003)

$$
\sigma_{2}^{*}=\left(\boldsymbol{\beta}^{\prime} W^{-1} \beta\right)^{-1} \boldsymbol{\beta}^{\prime} W^{-1} \mathbf{Y}_{[n]}=\sum_{r=1}^{n} a_{r} Y_{[r] r},
$$

where $\boldsymbol{\beta}=\left(\beta_{1}, \beta_{2}, \ldots, \beta_{n}\right)^{\prime}, W=\left[\operatorname{diag}\left(\lambda_{i}\right)\right]$ and $a_{r}=\frac{\beta_{r}}{\lambda_{r}} / \sum_{r=1}^{n} \frac{\beta_{r}^{2}}{\lambda_{r}}$. The variance of $\sigma_{2}^{*}$ is

$$
\operatorname{Var}\left[\sigma_{2}^{*}\right]=\left(\boldsymbol{\beta}^{\prime} W^{-1} \boldsymbol{\beta}\right)^{-1} \sigma_{2}^{2}=\frac{\sigma_{2}^{2}}{\sum_{r=1}^{n} \frac{\beta_{r}^{2}}{\lambda_{r}}} .
$$

Therefore, the relative efficiency of $\hat{\sigma}_{2, \mathrm{RSS}}$ to $\sigma_{2}^{*}$ is given by

$$
e_{2}=e\left(\sigma_{2}^{*} \mid \hat{\sigma}_{2, \mathrm{RSS}}\right)=\frac{4-\pi}{n \pi}\left(1-b_{n}\right) \sum_{r=1}^{n} \frac{\beta_{r}^{2}}{\lambda_{r}} .
$$

We can also provided a ranked set sample of size $n$ by each sample measurement of $Y$ which is taken on the unit that has the maximum value for the $X$ variable. Let $Y_{[n] r}$ be concomitants of largest order statistics $X_{(n) r}$ of the ${ }_{r}$ th sample for $r=1,2, \ldots, n$. Then, we call the collection of observations $X_{(n) r}$ as the upper ranked set sample (URSS). We can derive BLUE of $\sigma_{2}$ based on the observations URSS. From (2.3), the mean and variance of $Y_{[n] r}$ are given as $\sigma_{2} \beta$, and $\operatorname{Var}\left[y_{[n] r}\right]=\sigma_{2}^{2} \lambda_{n}$, respectively. Also, for $1 \leq r<s \leq n, \operatorname{Cov}\left[Y_{[n] r}, Y_{[n] s}\right]=0$. A BLUE for $\sigma_{2}$ based on URSS is obtained as $\tilde{\sigma}_{2}=\frac{1}{n \beta_{n}} \sum_{r=1}^{n} Y_{[n] r}$, and its variance is given by

$$
\operatorname{Var}\left(\tilde{\sigma}_{2}\right)=\frac{\sigma_{2}^{2} \lambda_{n}}{n \beta_{n}^{2}} \text {. }
$$

The efficiency of $\sigma_{2}^{*}$ relative to $\tilde{\sigma}_{2}$ and the efficiency of $\hat{\sigma}_{2, \mathrm{RSS}}$ relative to $\tilde{\sigma}_{2}$ are

$$
e_{3}=e\left(\tilde{\sigma}_{2} \mid \sigma_{2}^{*}\right)=\frac{n \beta_{n}^{2}}{\lambda_{n} \sum_{r=1}^{n} \frac{\beta_{r}^{2}}{\lambda_{r}}}, e_{4}=e\left(\tilde{\sigma}_{2} \mid \hat{\sigma}_{2, \mathrm{RSS}}\right)=\frac{(4-\pi)\left(1-b_{n}\right) n \beta_{n}^{2}}{n \pi \lambda_{n}}
$$

We have computed the values of $e_{2}, e_{3}$ and $e_{4}$ for $n=2(2) 10(5) 25$, and $\alpha= \pm .25, \pm .5, \pm .75, \pm 1$ in Table 1 . It can be seen that $\sigma_{2}^{*}$ is more efficient than $\hat{\sigma}_{2, \mathrm{Rss}}$ and for fixed $n \geq 2$, the efficiency increases with respect to $|\alpha|$. We can easily see that $\tilde{\sigma}_{2}$ is relatively more efficient than $\sigma_{2}^{*}$ and $\hat{\sigma}_{2, \mathrm{RSS}}$ for $0<\alpha \leq 1$. Also, $e_{3}$ and $e_{4}$ increases (decreases) with $n$ and $0<\alpha \leq 1 \quad(-1 \leq \alpha<0)$. Thus, we conclude that $\sigma_{2}^{*}$ and $\hat{\sigma}_{2, \mathrm{RSS}}$ are relatively more efficient than $\tilde{\sigma}_{2}$ when $-1 \leq \alpha<0$. 
Remark 2.1 Our assumption is that $\alpha$ is known, but sometimes $\alpha$ may not be known.

We know that the correlation coefficient between $X$ and $Y$ in MTBRD is $\frac{\alpha \pi(3-2 \sqrt{2})}{2(4-\pi)}$.

So by using the sample correlation coefficient q of the RSS observations $\left(X_{(r) r}, Y_{[r] r}\right)$, an estimator for $\alpha$ is

$$
\hat{\alpha}= \begin{cases}-1 & q<\frac{-\pi(3-2 \sqrt{2})}{2(4-\pi)} \\ \frac{2 q(4-\pi)}{\pi(3-\sqrt{2})} & \frac{-\pi(3-2 \sqrt{2})}{2(4-\pi)} \leq q \leq \frac{\pi(3-2 \sqrt{2})}{2(4-\pi)} \\ 1 & \frac{\pi(3-2 \sqrt{2})}{2(4-\pi)}<q .\end{cases}
$$

Table 1: The values of relative efficiencies $e_{2}, e_{3}$, and $e_{4}$ in MTBRD

\begin{tabular}{|c|c|c|c|c|c|c|c|c|c|}
\hline$n$ & $\alpha$ & $e_{2}$ & $e_{3}$ & $e_{4}$ & $n$ & $\alpha$ & $e_{2}$ & $e_{3}$ & $e_{4}$ \\
\hline \multirow[t]{8}{*}{2} & -1.00 & 1.0123 & 0.7805 & 0.7901 & \multirow[t]{8}{*}{10} & -1.00 & 1.0413 & 0.5944 & 0.6189 \\
\hline & -0.75 & 1.0069 & 0.8345 & 0.8402 & & -0.75 & 1.0201 & 0.6730 & 0.6865 \\
\hline & -0.50 & 1.0031 & 0.8892 & 0.8919 & & -0.50 & 1.0081 & 0.7657 & 0.7719 \\
\hline & -0.25 & 1.0008 & 0.9445 & 0.9452 & & -0.25 & 1.0019 & 0.8739 & 0.8755 \\
\hline & 0.25 & 1.0008 & 1.0555 & 1.0563 & & 0.25 & 1.0019 & 1.1484 & 1.1505 \\
\hline & 0.50 & 1.0031 & 1.1108 & 1.1141 & & 0.50 & 1.0081 & 1.3257 & 1.3365 \\
\hline & 0.75 & 1.0069 & 1.1655 & 1.1736 & & 0.75 & 1.0201 & 1.5433 & 1.5743 \\
\hline & 1.00 & 1.0123 & 1.2195 & 1.2345 & & 1.00 & 1.0413 & 1.8203 & 1.8955 \\
\hline \multirow[t]{8}{*}{4} & -1.00 & 1.0266 & 0.6612 & 0.6787 & \multirow[t]{8}{*}{15} & -1.00 & 1.0456 & 0.5817 & 0.6081 \\
\hline & -0.75 & 1.0138 & 0.7356 & 0.7457 & & -0.75 & 1.0219 & 0.6593 & 0.6736 \\
\hline & -0.50 & 1.0058 & 0.8164 & 0.8211 & & -0.50 & 1.0088 & 0.7538 & 0.7603 \\
\hline & -0.25 & 1.0014 & 0.9043 & 0.9055 & & -0.25 & 1.0021 & 0.8663 & 0.8681 \\
\hline & 0.25 & 1.0014 & 1.1047 & 1.1062 & & 0.25 & 1.0021 & 1.1603 & 1.1627 \\
\hline & 0.50 & 1.0058 & 1.2196 & 1.2266 & & 0.50 & 1.0088 & 1.3564 & 1.3683 \\
\hline & 0.75 & 1.0138 & 1.3465 & 1.3650 & & 0.75 & 1.0219 & 1.6044 & 1.6394 \\
\hline & 1.00 & 1.0266 & 1.4877 & 1.5272 & & 1.00 & 1.0456 & 1.9342 & 2.0223 \\
\hline \multirow[t]{8}{*}{6} & -1.00 & 1.0340 & 0.6228 & 0.6439 & \multirow[t]{8}{*}{20} & -1.00 & 1.0479 & 0.5758 & 0.6033 \\
\hline & -0.75 & 1.0170 & 0.7009 & 0.7128 & & -0.75 & 1.0228 & 0.6525 & 0.6673 \\
\hline & -0.50 & 1.0070 & 0.7889 & 0.7944 & & -0.50 & 1.0091 & 0.7477 & 0.7544 \\
\hline & -0.25 & 1.0017 & 0.8881 & 0.8895 & & -0.25 & 1.0021 & 0.8624 & 0.8642 \\
\hline & 0.25 & 1.0017 & 1.1271 & 1.1290 & & 0.25 & 1.0021 & 1.1667 & 1.1691 \\
\hline & 0.50 & 1.0070 & 1.2730 & 1.2819 & & 0.50 & 1.0091 & 1.3731 & 1.3855 \\
\hline & 0.75 & 1.0170 & 1.4428 & 1.4673 & & 0.75 & 1.0228 & 1.6384 & 1.6757 \\
\hline & 1.00 & 1.0340 & 1.6445 & 1.7004 & & 1.00 & 1.0479 & 1.9999 & 2.0956 \\
\hline \multirow[t]{8}{*}{8} & -1.00 & 1.0384 & 0.6047 & 0.6278 & \multirow[t]{8}{*}{25} & -1.00 & 1.0492 & 0.5725 & 0.6007 \\
\hline & -0.75 & 1.0189 & 0.6834 & 0.6963 & & -0.75 & 1.0233 & 0.6484 & 0.6635 \\
\hline & -0.50 & 1.0077 & 0.7746 & 0.7805 & & -0.50 & 1.0092 & 0.7440 & 0.7509 \\
\hline & -0.25 & 1.0018 & 0.8793 & 0.8809 & & -0.25 & 1.0021 & 0.8600 & 0.8619 \\
\hline & 0.25 & 1.0018 & 1.1400 & 1.1421 & & 0.25 & 1.0021 & 1.1706 & 1.1731 \\
\hline & 0.50 & 1.0077 & 1.3047 & 1.3147 & & 0.50 & 1.0092 & 1.3835 & 1.3963 \\
\hline & 0.75 & 1.0189 & 1.5026 & 1.5309 & & 0.75 & 1.0233 & 1.6600 & 1.6988 \\
\hline & 1.00 & 1.0384 & 1.7475 & 1.8146 & & 1.00 & 1.0492 & 2.0427 & 2.1434 \\
\hline
\end{tabular}




\section{Estimating based on ERSS and MERSS}

In this section, first we derive different estimators for $\sigma_{2}$ based on ERSS method with concomitant variable. This method introduced by Samawi et al. (1996) and can be described as follows:

Step 1. Select $n$ random samples each of size $n$ bivariate units from the population.

Step 2. If the sample size $n$ is even, then select from $\frac{n}{2}$ samples the smallest ranked unit $X$ together with the associated $Y$ and from the other $\frac{n}{2}$ samples the largest ranked unit $X$ together with the associated $Y$.This selected observations $\left(X_{(1) 1}, Y_{[1] 1}\right),\left(X_{(n) 2}, Y_{[n] 2}\right),\left(X_{(1) 3}, Y_{[1] 3}\right), \ldots,\left(X_{(1) n-1}, Y_{[1] n-1}\right),\left(X_{(n) n}, Y_{[n] n}\right)$ can be denoted by $\mathrm{ERSS}_{1}$.

Step 3. If $n$ is odd then select from $\frac{n-1}{2}$ samples the smallest ranked unit $X$ together with the associated $Y$ and from the other $\frac{n-1}{2}$ samples the largest ranked unit $X$ together with the associated $Y$ and from one sample the median of the sample for actual measurement. In this case the selected observations $\left(X_{(1) 1}, Y_{[1] 1}\right),\left(X_{(n) 2}, Y_{[n] 2}\right),\left(X_{(1) 3}, Y_{[1] 3}\right), \ldots,\left(X_{(n) n-1}, Y_{[n] n-1}\right), \quad\left(\frac{X_{(1) n}+X_{(n) n}}{2}, \frac{Y_{[1] n}+Y_{[n] n}}{2}\right) \quad$ can be denoted ERSS $_{2}$ and $\left(X_{(1) 1}, Y_{[1] 1}\right),\left(X_{(n) 2}, Y_{[n] 2}\right),\left(X_{(1) 3}, Y_{[1] 3}\right)$, $\ldots,\left(X_{(n) n-1}, Y_{[n] n-1}\right),\left(X_{\left(\frac{n+1}{2}\right) n}, Y_{\left[\frac{n+1}{2}\right] n}\right)$ can be denoted by ERSS $_{3}$.

Theorem 3.1 $i$. When $n$ is even, an unbiased estimator for $\sigma_{2}$ using ERSS is $_{1}$

$$
\hat{\sigma}_{2, \mathrm{ERSS}_{1}}=\frac{1}{n \sqrt{\frac{\pi}{2}}} \sum_{r=1}^{n / 2}\left(Y_{[1] 2 r-1}+Y_{[n] 2 r}\right),
$$

with the variance

$$
\operatorname{Var}\left(\hat{\sigma}_{2, \mathrm{ERSS}_{1}}\right)=\frac{\sigma_{2}^{2}(4-\pi)}{n \pi}\left(1-c_{n}\right),
$$

where $c_{n}=\frac{\pi}{2(4-\pi)}\left(\frac{\alpha(n-1)(1-\sqrt{2})}{n+1}\right)^{2}$. 
ii. When $n$ is odd, unbiased estimators for $\sigma_{2}$ using ERSS $_{2}$ and ERSS $_{3}$ are

$$
\begin{aligned}
\hat{\sigma}_{2, \mathrm{ERSS}_{2}}= & \frac{1}{n \sqrt{\frac{\pi}{2}}}\left(Y_{[1] 1}+Y_{[n] 2}+Y_{[1] 3}+\ldots+Y_{[n] n-1}+\frac{1}{2} Y_{[1] n}+\frac{1}{2} Y_{[n] n}\right), \\
\hat{\sigma}_{2, \mathrm{ERSS}_{3}}= & \frac{1}{n \sqrt{\frac{\pi}{2}}}\left(Y_{[1] 1}+Y_{[n] 2}+Y_{[1] 3}+\ldots+Y_{[n] n-1}+Y_{\left[\frac{n+1}{2}\right] n}\right) .
\end{aligned}
$$

with the variances

$$
\begin{aligned}
& \operatorname{Var}\left(\hat{\sigma}_{2, \mathrm{ERSS}_{2}}\right)=\frac{\sigma_{2}^{2}(4-\pi)}{n \pi}\left[\frac{2 n-1}{2 n}\left(1-4 c_{n}(n-1) / n\right)-d_{n}\right], \\
& \operatorname{Var}\left(\hat{\sigma}_{2, \mathrm{ERSS}_{3}}\right)=\frac{\sigma_{2}^{2}(4-\pi)}{n \pi}\left[1-4 c_{n}(n-1) / n\right],
\end{aligned}
$$

respectively, where $d_{n}=\frac{\pi(3-2 \sqrt{2})\left(n^{2}-3\right)}{2(4-\pi)(n+1)^{2}(n+2)} \alpha^{2}$.

Proof. The proof is obvious.

The efficiency of $\hat{\sigma}_{2, \mathrm{RSS}}$ relative to the estimators $\hat{\sigma}_{2, \mathrm{ERSS}}, \hat{\sigma}_{2, \mathrm{ERSS}_{2}}$ and $\hat{\sigma}_{2, \mathrm{ERSS}_{3}}$, respectively, are

$$
\begin{aligned}
& e_{5}=e\left(\hat{\sigma}_{2, \mathrm{ERSS}_{1}} \mid \hat{\sigma}_{2, \mathrm{RSS}}\right)=\frac{1-b_{n}}{1-c_{n}}, \\
& e_{6}=e\left(\hat{\sigma}_{2, \mathrm{ERSS}_{2}} \mid \hat{\sigma}_{2, \mathrm{RSS}}\right)=\frac{1-b_{n}}{\left.\frac{2 n-1}{2 n}\left(1-4 c_{n}(n-1) / n\right)-d_{n}\right)}, \\
& e_{7}=e\left(\hat{\sigma}_{2, \mathrm{ERSS}_{3}} \mid \hat{\sigma}_{2, \mathrm{RSS}}\right)=\frac{1-b_{n}}{1-4 c_{n}(n-1) / n} .
\end{aligned}
$$

Note that $1 \leq e_{i} \leq \frac{4}{3}$ for $i=5,6,7$. Also, for fixed $n, e_{i}$ 's increase in $|\alpha|$, and for fixed $|\alpha|, e_{i}$ 's increase in $n$. Therefore, $\hat{\sigma}_{2, \mathrm{ERSS}_{1}}, \hat{\sigma}_{2, \mathrm{ERSS}_{2}}$ and $\hat{\sigma}_{2, \mathrm{ERSS}_{3}}$ are more efficient than $\hat{\sigma}_{2, \mathrm{RSS}}$.

The concept of MERSS with concomitant variable is proposed by Al-Saleh and AlAnanbeh (2007) for estimation of means of the bivariate normal distribution. Here, we consider that the random vector $(X, Y)$ has a MTBRD as defined in (1.1). The procedure of MERSS with concomitant variable in MTBRD is as follows: 
Step 1. Select $n$ samples each of size $n$ from MTBRD using SRS. Identify by judgment the minimum of each sample with respect to the variable $X$.

Step 2. Repeat step 1, but for the maximum.

Note that the $2 n$ pairs of set $\left\{\left(X_{(1) r}, Y_{[1] r}\right),\left(X_{(n) r}, Y_{[n] r}\right) ; r=1,2, \ldots, n\right\}$ that are obtained using the above procedure, are independent but not identically distributed.

Theorem 3.2 An unbiased estimator of $\sigma_{2}$ based on MERSS is given by

$$
\hat{\sigma}_{2, \mathrm{MERSS}}=\frac{1}{n \sqrt{2 \pi}} \sum_{r=1}^{n}\left(Y_{[1] r}+Y_{[n] r}\right) \text {, }
$$

with the variance

$$
\operatorname{Var}\left(\hat{\sigma}_{2, \mathrm{MERSS}}\right)=\frac{\sigma_{2}^{2}(4-\pi)}{n \pi}\left(\frac{1-c_{n}}{2}\right) .
$$

Proof. The proof is obvious.

The efficiency of $\hat{\sigma}_{2, \mathrm{RSS}}$ relative to $\hat{\sigma}_{2, \mathrm{MERSS}}$ is

$$
e_{8}=e\left(\hat{\sigma}_{2, \mathrm{MERSS}} \mid \hat{\sigma}_{2, \mathrm{RSS}}\right)=\frac{2\left(1-b_{n}\right)}{1-c_{n}} \text {. }
$$

Note that $1 \leq e_{8} \leq \frac{8}{3}$. Thus, $\hat{\sigma}_{2, \mathrm{MERSS}}$ is more efficient than $\hat{\sigma}_{2, \mathrm{RSS}}$. Also, the efficiency of $\hat{\sigma}_{2, \mathrm{MERSS}}$ relative to $\sigma_{2}^{*}$ and $\tilde{\sigma}_{2}$ are

$$
\begin{aligned}
& e_{9}=e\left(\sigma_{2}^{*} \mid \hat{\sigma}_{2, \mathrm{MERSS}}\right)=\frac{4-\pi}{n \pi}\left(\frac{1-c_{n}}{2}\right) \sum_{r=1}^{n} \frac{\beta_{r}^{2}}{\lambda_{r}}, \\
& e_{10}=e\left(\tilde{\sigma}_{2} \mid \hat{\sigma}_{2, \mathrm{MERSS}}\right)=\frac{(4-\pi) \beta_{n}^{2}}{\pi \lambda_{n}}\left(\frac{1-c_{n}}{2}\right) .
\end{aligned}
$$

The efficiency of $\sigma_{2}^{*}$ relative to the estimators $\hat{\sigma}_{2, \mathrm{ERSS}_{1}}, \hat{\sigma}_{2, \mathrm{ERSS}_{2}}$ and $\hat{\sigma}_{2, \mathrm{ERSS}_{3}}$ are

$$
\begin{aligned}
& e_{11}=e\left(\hat{\sigma}_{2, \mathrm{ERSS}_{1}} \mid \sigma_{2}^{*}\right)=\frac{n \pi}{(4-\pi) \sum_{r=1}^{n} \frac{\beta_{r}^{2}}{\lambda_{r}}\left(1-c_{n}\right)}, \\
& e_{12}=e\left(\hat{\sigma}_{2, \mathrm{ERSS}_{2}} \mid \sigma_{2}^{*}\right)=\frac{n \pi}{(4-\pi) \sum_{r=1}^{n} \frac{\beta_{r}^{2}}{\lambda_{r}}\left[\frac{2 n-1}{2 n}\left(1-4 c_{n}\right)-d_{n}\right]}, \\
& e_{13}=e\left(\hat{\sigma}_{2, \mathrm{ERSS}_{3}} \mid \sigma_{2}^{*}\right)=\frac{n \pi}{(4-\pi) \sum_{r=1}^{n} \frac{\beta_{r}^{2}}{\lambda_{r}}\left(1-\frac{4(n-1)}{n} d_{n}\right)} .
\end{aligned}
$$


Finally, the efficiency of $\tilde{\sigma}_{2}$ relative to $\hat{\sigma}_{2, \mathrm{ERSS}_{1}}, \hat{\sigma}_{2, \mathrm{ERSS}}$ and $\hat{\sigma}_{2, \mathrm{ERSS}}$ are

$$
\begin{aligned}
& e_{14}=e\left(\hat{\sigma}_{2, \mathrm{ERSS}_{1}} \mid \tilde{\sigma}_{2}\right)=\frac{\pi \lambda_{n}}{(4-\pi) \beta_{n}^{2}\left(1-c_{n}\right)}, \\
& e_{15}=e\left(\hat{\sigma}_{2, \mathrm{ERSS}_{2}} \mid \tilde{\sigma}_{2}\right)=\frac{\pi \lambda_{n}}{(4-\pi) \beta_{n}^{2}\left[\frac{2 n-1}{2 n}\left(1-4 c_{n}\right)-d_{n}\right]}, \\
& e_{16}=e\left(\hat{\sigma}_{2, \mathrm{ERSS}_{3}} \mid \tilde{\sigma}_{2}\right)=\frac{\pi \lambda_{n}}{(4-\pi) \beta_{n}^{2}\left(1-\frac{4(n-1)}{n} c_{n}\right)} .
\end{aligned}
$$

We computed the values of $e_{j}, j=9,10, \ldots, 16$ for $\alpha= \pm 0.25, \pm 0.5, \pm 0.75, \pm 1$ and $n=5(5) 20$. The results are given in Table 2, and we can conclude that

i) The efficiencies of $\hat{\sigma}_{2, \text { MERSS }}$ relative to $\sigma_{2}^{*}$ and $\tilde{\sigma}_{2}$ are less than 1 for $n \geq 5$. So, $\hat{\sigma}_{2, \text { MERSS }}$ is relatively more efficient than $\sigma_{2}^{*}$ and $\tilde{\sigma}_{2}$.

ii) The efficiencies of $\sigma_{2}^{*}$ relative to the estimators $\hat{\sigma}_{2, \mathrm{ERSS}}, \hat{\sigma}_{2, \mathrm{ERSS}}$ and $\hat{\sigma}_{2, \mathrm{ERSS}}$ are more than 1 for $n \geq 5$. Thus, $\hat{\sigma}_{2, \mathrm{ERSS}_{1}}, \hat{\sigma}_{2, \mathrm{ERSS}}$ and $\hat{\sigma}_{2, \mathrm{ERSS}_{3}}$ are relatively more efficient than $\sigma_{2}^{*}$.

iii) The efficiencies of $\tilde{\sigma}_{2}$ relative to the estimators $\hat{\sigma}_{2, \mathrm{ERSS}}, \hat{\sigma}_{2, \mathrm{ERSS}}$ and $\hat{\sigma}_{2, \mathrm{ERSS}}$ are more than (less than) 1 for $-1 \leq \alpha<O(0<\alpha \leq 1)$ and $n \geq 5$. Thus $\tilde{\sigma}_{2}$ is relatively more efficient than $\hat{\sigma}_{2, \mathrm{ERSS}}, \hat{\sigma}_{2, \mathrm{ERSS}}$ and $\hat{\sigma}_{2, \mathrm{ERSS}_{3}}$ when $0<\alpha \leq 1$.

\section{Estimating based on DRERSS}

In this section, first we obtain different estimators for $\sigma_{2}$ based on DRERSS method with concomitant variable. This method introduced by Al-Omari (2011) and can be described as follows:

Step 1. Select $n^{2}$ random samples each of size $n$ bivariate units from the population.

Step 2. Select the coefficient $k=[\beta n]$, where $0<\beta<1$, and $[x]$ is the largest integer value less than or equal to $x$. 
Table 2: The values of $e_{j}$ for $j=9,10, \ldots, 16$ in MTBRD

\begin{tabular}{|c|c|c|c|c|c|c|c|c|c|}
\hline$n$ & $\alpha$ & $e_{9}$ & $e_{10}$ & $e_{11}$ & $e_{12}$ & $e_{13}$ & $e_{14}$ & $e_{15}$ & $e_{16}$ \\
\hline \multirow[t]{8}{*}{5} & -1.00 & 0.4688 & 0.3719 & 1.0545 & 1.1708 & 1.0190 & 1.6530 & 1.8354 & 1.5974 \\
\hline & -0.75 & 0.4830 & 0.3540 & 1.0293 & 1.1432 & 1.0109 & 1.4400 & 1.5994 & 1.4142 \\
\hline & -0.50 & 0.4926 & 0.3401 & 1.0126 & 1.1249 & 1.0049 & 1.2656 & 1.4060 & 1.2559 \\
\hline & -0.25 & 0.4981 & 0.3285 & 1.0031 & 1.1145 & 1.0012 & 1.1211 & 1.2456 & 1.1190 \\
\hline & 0.25 & 0.4981 & 0.3086 & 1.0031 & 1.1145 & 1.0012 & 0.8975 & 0.9971 & 0.8958 \\
\hline & 0.50 & 0.4926 & 0.2990 & 1.0126 & 1.1249 & 1.0049 & 0.8100 & 0.8998 & 0.8038 \\
\hline & 0.75 & 0.4830 & 0.2891 & 1.0293 & 1.1432 & 1.0109 & 0.7346 & 0.8160 & 0.7215 \\
\hline & 1.00 & 0.4688 & 0.2782 & 1.0545 & 1.1708 & 1.0190 & 0.6694 & 0.7432 & 0.6469 \\
\hline \multirow[t]{8}{*}{10} & -1.00 & 0.4398 & 0.3942 & 1.1237 & 1.1828 & 1.0924 & 1.8906 & 1.9900 & 1.8378 \\
\hline & -0.75 & 0.4674 & 0.3656 & 1.0636 & 1.1195 & 1.0485 & 1.5804 & 1.6635 & 1.5580 \\
\hline & -0.50 & 0.4859 & 0.3461 & 1.0266 & 1.0806 & 1.0206 & 1.3407 & 1.4112 & 1.3328 \\
\hline & -0.25 & 0.4965 & 0.3310 & 1.0064 & 1.0594 & 1.0050 & 1.1516 & 1.2123 & 1.1500 \\
\hline & 0.25 & 0.4965 & 0.3064 & 1.0064 & 1.0594 & 1.0050 & 0.8764 & 0.9225 & 0.8751 \\
\hline & 0.50 & 0.4859 & 0.2946 & 1.0266 & 1.0806 & 1.0206 & 0.7744 & 0.8151 & 0.7698 \\
\hline & 0.75 & 0.4674 & 0.2818 & 1.0636 & 1.1195 & 1.0485 & 0.6892 & 0.7254 & 0.6794 \\
\hline & 1.00 & 0.4398 & 0.2668 & 1.1237 & 1.1828 & 1.0924 & 0.6173 & 0.6497 & 0.6001 \\
\hline \multirow[t]{8}{*}{15} & -1.00 & 0.4266 & 0.4051 & 1.1592 & 1.1992 & 1.1333 & 1.9930 & 2.0617 & 1.9485 \\
\hline & -0.75 & 0.4604 & 0.3706 & 1.0801 & 1.1173 & 1.0681 & 1.6384 & 1.6948 & 1.6202 \\
\hline & -0.50 & 0.4829 & 0.3484 & 1.0331 & 1.0687 & 1.0284 & 1.3706 & 1.4178 & 1.3644 \\
\hline & -0.25 & 0.4958 & 0.3320 & 1.0079 & 1.0427 & 1.0068 & 1.1634 & 1.2036 & 1.1622 \\
\hline & 0.25 & 0.4958 & 0.3056 & 1.0079 & 1.0427 & 1.0068 & 0.8686 & 0.8986 & 0.8677 \\
\hline & 0.50 & 0.4829 & 0.2929 & 1.0331 & 1.0687 & 1.0284 & 0.7616 & 0.7879 & 0.7582 \\
\hline & 0.75 & 0.4604 & 0.2789 & 1.0801 & 1.1173 & 1.0681 & 0.6732 & 0.6964 & 0.6658 \\
\hline & 1.00 & 0.4266 & 0.2621 & 1.1592 & 1.1992 & 1.1333 & 0.5993 & 0.6200 & 0.5859 \\
\hline \multirow[t]{8}{*}{20} & -1.00 & 0.4191 & 0.4115 & 1.1805 & 1.2107 & 1.1587 & 2.0501 & 2.1026 & 2.0123 \\
\hline & -0.75 & 0.4564 & 0.3734 & 1.0896 & 1.1176 & 1.0795 & 1.6700 & 1.7128 & 1.6550 \\
\hline & -0.50 & 0.4812 & 0.3497 & 1.0368 & 1.0633 & 1.0330 & 1.3866 & 1.4222 & 1.3816 \\
\hline & -0.25 & 0.4953 & 0.3325 & 1.0088 & 1.0346 & 1.0079 & 1.1697 & 1.1997 & 1.1687 \\
\hline & 0.25 & 0.4953 & 0.3052 & 1.0088 & 1.0346 & 1.0079 & 0.8646 & 0.8868 & 0.8639 \\
\hline & 0.50 & 0.4812 & 0.2920 & 1.0368 & 1.0633 & 1.0330 & 0.7551 & 0.7744 & 0.7523 \\
\hline & 0.75 & 0.4564 & 0.2773 & 1.0896 & 1.1176 & 1.0795 & 0.6650 & 0.6821 & 0.6591 \\
\hline & 1.00 & 0.4191 & 0.2595 & 1.1805 & 1.2107 & 1.1587 & 0.5902 & 0.6054 & 0.5794 \\
\hline
\end{tabular}

Step 3. If $n$ is even, from the first $\frac{n^{2}}{2}$ samples select the $(k+1)$ th smallest unit $X$ together with the associated $Y$ and from the second $\frac{n^{2}}{2}$ samples the $(n-k)$ th smallest unit $X$ together with the associated $Y$. If $n$ is odd, select from the first $\frac{n(n-1)}{2}$ samples the $(k+1)$ th smallest unit $X$ together with the associated $Y$, and from the next $n$ samples the $\frac{n+1}{2}$ th smallest unit $X$ together with the associated $Y$, and from the last 
$\frac{n(n-1)}{2}$ samples the $(n-k)$ th smallest ranked unit with the associated $Y$. This step yield $n$ samples each of size $n$.

Step 4. For the $n$ samples obtained in Step 3, if $n$ is even, select for actual measurement from the first $\frac{n}{2}$ samples the $(k+1)$ th smallest ranked unit $X$ together with the associated $Y$ and from the second $\frac{n}{2}$ samples the $(n-k)$ th smallest ranked unit $X$ together with the associated $Y$. If $n$ is odd, select from the first $\frac{n-1}{2}$ samples the $(k+1)$ th smallest ranked unit $X$ together with the associated $Y$, the median from the next sample and from the last $\frac{n-1}{2}$ samples the $(n-k)$ th smallest ranked unit $X$ together with the associated $Y$. This step yields one sample of size $n$ units from the DRERSS data.

Theorem 4.1 $i$. When $n$ is even, an unbiased estimator for $\sigma_{2}$ using DRERSS is

$$
\hat{\sigma}_{2, \mathrm{DRERSSE}}=\frac{1}{n \sqrt{\frac{\pi}{2}}}\left[\sum_{r=1}^{n / 2} Y_{[k+1] r}+\sum_{r=(n+2) / 2}^{n} Y_{[n-k] r}\right]
$$

with the variance

$$
\operatorname{Var}\left(\hat{\sigma}_{2, \mathrm{DRERSSE}}\right)=\frac{\sigma_{2}^{2}(4-\pi)}{n \pi}\left(1-w_{n}\right),
$$

where $w_{n}=\frac{\pi}{2(4-\pi)}\left(\frac{\alpha(n-2 k-1)(1-\sqrt{2})}{n+1}\right)^{2}$.

ii. When $n$ is odd, an unbiased estimator for $\sigma_{2}$ using DRERSS is

$$
\hat{\sigma}_{2, \text { DRERSSO }}=\frac{1}{n \sqrt{\frac{\pi}{2}}}\left[\sum_{r=1}^{(n-1) / 2} Y_{[k+1] r}+Y_{\left[\frac{n+1}{2}\right] r}+\sum_{r=(n+3) / 2}^{n} Y_{[n-k] r}\right]
$$

with the variance

$$
\operatorname{Var}\left(\hat{\sigma}_{2, \mathrm{DRERSSO}}\right)=\frac{\sigma_{2}^{2}(4-\pi)}{n \pi}\left(1-z_{n}\right),
$$

where $z_{n}=\frac{n-1}{n} w_{n}$.

Proof. The proof is obvious. 
The efficiency of $\hat{\sigma}_{2, \mathrm{RSS}}$ relative to the estimator $\hat{\sigma}_{2, \mathrm{DRERSS}}$ is

$$
e_{17}=e\left(\hat{\sigma}_{2, \mathrm{DRERSS}} \mid \hat{\sigma}_{2, \mathrm{RSS}}\right)= \begin{cases}\frac{1-b_{n}}{1-w_{n}} & n \text { is even } \\ \frac{1-b_{n}}{1-z_{n}} & n \text { is odd }\end{cases}
$$

Note that $1 \leq e_{17} \leq 1.3$. Thus, $\hat{\sigma}_{2, \text { DRERSS }}$ is more efficient than $\hat{\sigma}_{2, \mathrm{RSS}}$. The efficiency of $\hat{\sigma}_{2, \text { DRERSS }}$ relative to the estimator $\hat{\sigma}_{2, \text { MERSS }}$ is

$$
e_{18}=e\left(\hat{\sigma}_{2, \text { MERSS }} \mid \hat{\sigma}_{2, \text { DRERSS }}\right)= \begin{cases}\frac{2\left(1-w_{n}\right)}{1-c_{n}} & n \text { is even } \\ \frac{2\left(1-z_{n}\right)}{1-c_{n}} & n \text { is odd }\end{cases}
$$

Where $1 \leq e_{18} \leq 2$. So, $\hat{\sigma}_{2, \text { MERSS }}$ is more efficient than $\hat{\sigma}_{2, \text { DRERSS }}$. The efficiency of $\hat{\sigma}_{2, \text { DRERSS }}$ relative to the estimators $\sigma_{2}^{*}$ and $\tilde{\sigma}_{2}$ are

$$
\begin{gathered}
e_{19}=e\left(\sigma_{2}^{*} \mid \hat{\sigma}_{2, \mathrm{DRERSS}}\right)= \begin{cases}\frac{\left(1-w_{n}\right)(4-\pi)}{n \pi} \sum_{r=1}^{n} \frac{\beta_{r}^{2}}{\lambda_{r}} & n \text { is even } \\
\frac{\left(1-z_{n}\right)(4-\pi)}{n \pi} \sum_{r=1}^{n} \frac{\beta_{r}^{2}}{\lambda_{r}} & n \text { is odd }\end{cases} \\
e_{20}=e\left(\tilde{\sigma}_{2} \mid \hat{\sigma}_{2, \mathrm{DRERSS}}\right)= \begin{cases}\frac{\left(1-w_{n}\right)(4-\pi)}{n \pi} \times \frac{n \beta_{n}^{2}}{\lambda_{n}} & n \text { is odd } \\
\frac{\left(1-z_{n}\right)(4-\pi)}{n \pi} \times \frac{n \beta_{n}^{2}}{\lambda_{n}} & \end{cases}
\end{gathered}
$$

We computed the values of $e_{19}$ and $e_{20}$ for $\alpha= \pm 0.25, \pm 0.5, \pm 0.75, \pm 1, k=0,1,2,3$ and $n=4,5,6,7$. The results are given in Table 3 , and we can conclude that:

i. The efficiency of $\hat{\sigma}_{2, \text { DRERSS }}$ relative to $\sigma_{2}^{*}$ is less than 1 for $k=0$ and $n \geq 4$. So, $\hat{\sigma}_{2, \text { DRERSS }}$ is relatively more efficient than $\sigma_{2}^{*}$.

ii. The efficiency of $\hat{\sigma}_{2, \text { DRERSS }}$ relative to the $\sigma_{2}^{*}$ is more than 1 for $n \geq 5$ and $k=1,2,3$. So, $\sigma_{2}^{*}$ is relatively more than efficient than $\hat{\sigma}_{2, \text { DRERSS }}$.

iii. The efficiency of $\hat{\sigma}_{2, \text { DRERSS }}$ relative to the $\tilde{\sigma}_{2}$ is more than (less than) 1 for $0<\alpha \leq 1$ $(-1 \leq \alpha<0)$ and $n \geq 4, k \geq 0$. Thus $\hat{\sigma}_{2, \text { DRERSS }}$ is relatively more efficient than $\tilde{\sigma}_{2}$ when $-1 \leq \alpha<0$ and $n \geq 4, k \geq 0$. 
Table 3: The values of $e_{19}$ and $e_{20}$ in MTBRD

\begin{tabular}{|c|c|c|c|c|c|c|c|c|c|}
\hline & & & $k=0$ & & $k=1$ & & $k=2$ & & $k=3$ \\
\hline$n$ & $\alpha$ & $e_{19}$ & $e_{20}$ & $e_{19}$ & $e_{20}$ & $e_{19}$ & $e_{20}$ & $e_{19}$ & $e_{20}$ \\
\hline \multirow[t]{8}{*}{4} & -1.00 & 0.9576 & 0.6460 & 1.0661 & 0.7191 & 1.0661 & 0.7191 & 0.9576 & 0.6460 \\
\hline & -0.75 & 0.9768 & 0.7269 & 1.0357 & 0.7708 & 1.3578 & 0.7708 & 0.9768 & 0.7264 \\
\hline & -0.50 & 0.9898 & 0.8136 & 1.0154 & & 1.0154 & 0.8346 & 0.9898 & 0.8136 \\
\hline & -0.25 & 0.9975 & 0.9050 & 1.00 & 0.9107 & 1.003 & 0.9107 & 0.9975 & 0.9050 \\
\hline & 0.25 & & 2 & & & & & & 50 \\
\hline & 0.50 & 0.9898 & 1.1953 & 1.0154 & 1.2262 & 1.0154 & 1.2262 & 0.9898 & 1.1953 \\
\hline & 0.75 & 0.9768 & 1.2920 & 1.0357 & 1.3699 & 1.0357 & 1.3699 & 0.9768 & 1.2920 \\
\hline & 1.00 & 0.9576 & 1.3845 & 1.0661 & 1.5414 & 1.0661 & 1.5414 & 0.9576 & 1.3845 \\
\hline \multirow[t]{8}{*}{5} & -1.00 & & 0.6320 & & & 1.0 & 0.7114 & 1.0 & 0.6916 \\
\hline & -0.75 & & 0.7111 & & & & 38 & 18 & 0.7469 \\
\hline & -0.50 & 0.9923 & 0.7996 & 1.0136 & 8 & 1.0208 & 0.8226 & 1.0136 & 0.8168 \\
\hline & -0.25 & 0.9981 & 0.8962 & 1.0033 & 99 & 1.0051 & 0.9025 & 1.003 & 0.9009 \\
\hline & 0.25 & 0.9981 & 1.1101 & & & & & 033 & 1.1159 \\
\hline & 0.50 & & 1. & & & & & & 1.2521 \\
\hline & 0.75 & 824 & 0 & 1 & & 1.0 & 1. & 1. & 1.4136 \\
\hline & 1.00 & 0.9682 & 1.4695 & 1.0594 & 1.6080 & 1.0898 & & 1.0594 & 1.6080 \\
\hline \multirow[t]{8}{*}{6} & -1.00 & 5 & 0.5890 & 1.0339 & 0.6609 & 1.0902 & 0.6 & 1.0902 & 0.6968 \\
\hline & -0.75 & & & & & & & & \\
\hline & -0.50 & & 0.7800 & & & 1.0 & & 1.0 & 0.8112 \\
\hline & -0.25 & 0.9954 & 0.8873 & 1.0018 & 0.8930 & 1.0050 & 0.8959 & 1.0050 & 0.8959 \\
\hline & 0.25 & 0.9954 & 1.1159 & 1.0018 & 31 & 1.0050 & 1.1267 & 1.0050 & 1.1267 \\
\hline & 0.50 & 0.9814 & & & & 1.0 & & 07 & 1.2818 \\
\hline & 0.75 & & & & & & & & 1.4735 \\
\hline & 1.00 & & & & & 1.0 & & & 1.7153 \\
\hline \multirow[t]{8}{*}{7} & -1.00 & & 0.58 & 1.0287 & 0 . & 1.0843 & 0.6 & 1.1029 & 0.6948 \\
\hline & -0.75 & 0 & 067 & 19 & & 1.0448 & 0.7320 & 48 & 0.7390 \\
\hline & -0.50 & 0.9847 & 0.7747 & 1.0062 & 0.7916 & 1.0192 & 0.8018 & 1.0235 & 0.8051 \\
\hline & -0.25 & & & & & & & & 0.8916 \\
\hline & 0.25 & & & & & & & & 1.1343 \\
\hline & 0.5 & & & & & 1.0 & & & 1.3014 \\
\hline & 0.75 & & 1.38 & 1.0 & & 1.0 & 1.4 & & 1.5122 \\
\hline & 1.00 & 0.9359 & 1.5139 & 1.0287 & 1.6639 & 1.0843 & 1.7539 & 1.1029 & 1.7839 \\
\hline
\end{tabular}

\section{An application}

A reappraisal of caloric requirements in healthy women are done by Owen et al. (1986). The results of this study show that the body weight of women was highly related to the resting metabolic rate (RMR) of the women.

We considered a bivariate data set from the 44 women data such that the first component $X$ represents the body weight $(\mathrm{kg})$, and the second components $Y$ represents resting metabolic rate (RMR) $(\mathrm{kcal} / 24 \mathrm{hr})$. Clearly, the the body weight $(\mathrm{kg})$ can be measured very easily but the RMR is difficult to measure. We selected 6 random samples with size 
6 from 44 women data and ranked the sampling units of each sample according to the $X$ variate (body weight). We measureed the ranked set sample observations $Y_{[r] r}$ corresponding to $X_{(r) r}$. The obtained RSS, ERSS ${ }_{1}$ and MERSS observations are given in

Table 4. Since the sample correlation coefficient is $q>\frac{1}{3}$, the estimate for $\alpha$ is 1 (see Remark 2.1).

The computed values of $\hat{\sigma}_{2, \mathrm{RSS}}, \hat{\sigma}_{2, \mathrm{ERSS}_{1}}, \hat{\sigma}_{2, \mathrm{MERSS}}$ are 1142.57, 1009.32, and 979.40, respectively. We can find that the estimated values for $\sigma_{2}$ based on different samplings are close.

Table 4: Obtained RSS, ERSS ${ }_{1}$ and MERSS observations

\begin{tabular}{|c|c|cccccc|}
\hline & $r$ & 1 & 2 & 3 & 4 & 5 & 6 \\
\hline \multirow{2}{*}{ RSS } & $X_{(r) r}$ & 49.9 & 48.1 & 56 & 62.1 & 82 & 99.8 \\
& $Y_{[r] r}$ & 1079 & 1372 & 1392 & 1574 & 1536 & 1639 \\
\hline \multirow{2}{*}{ ERSS $_{1}$} & $X_{(1) 2 r-1}$ & 49.9 & 55 & 66.4 & & & \\
& $Y_{[1] 2 r-1}$ & 1079 & 1034 & 1205 & & & \\
\cline { 2 - 8 } & $X_{(n) 2 r}$ & 64.9 & 66 & 99.8 & & & \\
& $Y_{[n] 2 r}$ & 1365 & 1268 & 1639 & & & \\
\hline \multirow{7}{*}{ MERSS } & $X_{(1) r}$ & 49.9 & 43.1 & 55 & 59.2 & 66.4 & 83.4 \\
& $Y_{[1] r}$ & 1079 & 870 & 1034 & 1342 & 1205 & 1248 \\
\cline { 2 - 8 } & $X_{(n) r}$ & 61.4 & 64.9 & 59 & 66 & 82 & 99.8 \\
& $Y_{[n] r}$ & 1351 & 1365 & 1178 & 1268 & 1151 & 1639 \\
\hline
\end{tabular}

\section{References}

1. Al-Odat, M. and Al-Saleh, M.F. (2001). A variation of ranked set sampling. Journal of Applied Statistical Science, 10(2), 137-146.

2. Al-Omari, A.I. (2011). Estimation of mean based on modified robust extreme ranked set sampling. Journal of Statistical Computation and Simulation, 81(8), 1055-1066.

3. Al-Saleh, M. F. and Al-Ananbeh, A. M. (2005). Estimating the correlation coefficient in a bivariate normal distribution using moving extreme ranked set sampling with a concomitant variable. Journal of the Korean Statistical Society, 34(2), 125-140.

4. Al-Saleh, M. F. and Al-Ananbeh, A. M. (2007). Estimation of the means of the bivariate normal using moving extreme ranked set sampling with concomitant variable. Statistical Papers, 48(2), 179-195. 
5. Al-Saleh, M. F. and Diab, Y. A. (2009). Estimation of the parameters of Downton's bivariate exponential distribution using ranked set sampling scheme. Journal of Statistical Planning and Inference, 139(2), 277-286.

6. Bairamov, I. and Bekci, M. (1999). Concomitant of order statistics in fgm type bivariate uniform distributions. Istatistik, Journal of the Turkish Statistical Association, 2(2), 135-144.

7. Balasubramanian, K. and Beg, M. I. (1997). Concomitants of order statistics in Morgenstern type bivariate exponential distribution. Journal of Applied Statistical Science, 54(4), 233-245.

8. Chacko, M. and Thomas, P. Y. (2008). Estimation of a parameter of Morgenstern type bivariate exponential distribution by ranked set sampling. Annals of the Institute of Statistical Mathematics, 60(2), 301-318.

9. Chacko, M. and Thomas, P. Y. (2011). Estimation of parameter of Morgenstern type bivariate exponential distribution using concomitants of order statistics. Statistical Methodology, 8(4), 363-376.

10. David, H. A. and Nagaraja, H. (2003). Order Statistics. John Wiley and Sons.

11. D'Este, G. (1981). A Morgenstern-type bivariate gamma distribution. Biometrika, 68(1), 339-340.

12. Farlie, D. J. G. (1960). The performance of some correlation coefficients for a general bivariate distribution. Biometrika, 47(3/4), 307-323.

13. Gumbel, E. J. (1960). Bivariate exponential distributions. Journal of the American Statistical Association, 55(292), 698-707.

14. Gumbel, E. J. (1961). Bivariate logistic distributions. Journal of the American Statistical Association, 56(294), 335-349.

15. Lam, K., Sinha, B. K., and Wu, Z. (1994). Estimation of parameters in a twoparameter exponential distribution using ranked set sample. Annals of the Institute of Statistical Mathematics, 46(4), 723-736.

16. McIntyre, G. A. (1952). A method for unbiased selective sampling, using ranked sets. Australian Journal of Agricultural Research, 3(4): 385-390.

17. Morgenstern, D. (1956). Einfache beispiele zweidimensionaler verteilungen. Mitteilingsblatt für Mathematishe Statistik, 8(1), 234-235.

18. Owen, O. E., Kavle, E., Owen, R. S., Polansky, M., Caprio, S., Mozzoli, M. A., Kendrick, Z. V., Bushman, M., and Boden, G. (1986). A reappraisal of caloric requirements in healthy women. The American Journal of Clinical Nutrition, 44(1), 1-19.

19. Samawi, H. M., Ahmed, M. S., and Abu-Dayyeh, W. (1996). Estimating the population mean using extreme ranked set sampling. Biometrical Journal, 38(5): 577-586.

20. Scaria, J. and Nair, N. U. (1999). On concomitants of order statistics from Morgenstern family. Biometrical Journal, 41(4), 483-489. 
21. Singh, H. P and Mehta.V (2015). Estimation of scale parameter of a Morgenstern type bivariate uniform distribution using censored ranked set samples. Model Assisted Statistics and Applications, 10(2), 139-153.

22. Stokes, S. L. (1977). Ranked set sampling with concomitant variables. Communications in Statistics - Theory and Methods, 6(12), 1207-1211.

23. Stokes, S. L. (1980). Inferences on the correlation coefficient in bivariate normal populations from ranked set samples. Journal of the American Statistical Association, 75(372), 989-995.

24. Stokes, S. L. (1995). Parametric ranked set sampling. Annals of the Institute of Statistical Mathematics, 47(3), 465-482.

25. Tahmasebi, S. and Jafari, A. A. (2012). Estimation of a scale parameter of Morgenstern type bivariate uniform distribution by ranked set sampling. Journal of Data Science, 10, 129-141.

26. Tahmasebi, S. and Jafari, A. A. (2015). Concomitants of order statistics and record values from Morgenstern type bivariate generalized exponential distribution. Bulletin of the Malaysian Mathematical Sciences Society, 38(4), 1411-1423.

27. Tahmasebi, S. and Jafari, A. A. (2014). Estimators for the parameter mean of Morgenstern type bivariate generalized exponential distribution using ranked set sampling. Statistics and Operations Research Transactions, 38(2), 161-180.

28. Tahmasebi, S. and Jafari, A. A. (2015). A review on unbiased estimators of a parameter from Morgenstern type bivariate gamma distribution using ranked set sampling. Azerbaijan Journal of Mathematics, 5(2), 3-12. 